\title{
Optimization by Response Surface Methodology (RSM) of the Kharasch-Sosnovsky Oxidation of Valencene
}

\author{
Ana Leticia García-Cabeza, Lindsey P. Ray, Rubén Marín-Barrios, María J. Ortega, \\ F. Javier Moreno-Dorado, Francisco M. Guerra,* and Guillermo M. Massanet \\ Departamento de Química Orgánica, Facultad de Ciencias, Universidad de Cádiz, 11510 Puerto Real, Cádiz, Spain
}

Supporting Information

ABSTRACT: Response surface methodology (RSM) has been employed to model and optimize the Kharasch-Sosnovsky allylic oxidation of valencene. The detailed effects of the amounts of oxidant, substrate, copper catalyst, 1,8diazabicyclo[5.4.0] undec-7-ene (DBU), and temperature have been studied. The concentration of substrate and quantity of oxidant have been determined as the most significant variables. The interaction effects on the yield have been investigated using a three-level full-factorial design. The yield of benzoyloxyvalencene has been improved to $99 \%$. Analysis of variance (ANOVA) has been used to evaluate the goodness of fit of the model.

\section{INTRODUCTION}

The search for new allylic oxidation reactions is a very active area in organic synthesis. ${ }^{1}$ The presence of a double bond activates the allylic $\mathrm{C}-\mathrm{H}$ bond, enabling the selective installation of an oxygenated function. The discovery of new ways to accomplish this transformation allows streamlining the synthesis of complex molecules, as White et al. have demonstrated in the preparation of highly complex compounds from the corresponding alkenes. ${ }^{2}$ Recently, Hartwig et al. have demonstrated that the allylic oxidation of olefins is a useful method to extend the functionality along a carbon backbone by an iterative oxidation/homologation sequence. $^{3}$

Classically, these oxidations have been performed by a series of metal-catalyzed processes, involving metals such as chromium, manganese, rhodium, iron, copper, cobalt, or palladium. ${ }^{4}$ It is noteworthy the wide use of those methods based on selenium reagents, which involve an ene reaction and a further [2,3] rearrangement. Selenium dioxide in catalytic or stoichiometric amounts has been a reagent of reference given its reliability and predictability. ${ }^{5}$ It produces, however, important amounts of organoselenium byproducts along with reduced forms of selenium, difficult to remove.

An alternative to the aforementioned methods is the Kharasch-Sosnovsky reaction, which involves the oxidation of an alkene by a peroxyester in the presence of a copper or cobalt source providing the corresponding allylic ester. ${ }^{6}$ The reaction was first described in the 1950 s decade, ${ }^{7}$ and it remained unnoticed until the mid 1990s in which the Pfaltz, ${ }^{8}$ Katsuki, ${ }^{9}$ and Andrus $^{10}$ groups developed enantioselective versions of the reaction.

Allylic oxidation is particularly attractive in terpene synthesis. These compounds exhibit remarkable biological activities, and their preparation is of high economic interest. Their synthesis is generally marked by the presence of multiple oxygenated functions in a relatively small carbon skeleton, which requires the employment of selective oxidation methods after the assembly of the carbon framework. ${ }^{11}$
In the course of our research on the synthesis of thapsigargins, a family of sesquiterpene lactones with remarkable anti SERCAATPase activity, ${ }^{12}$ the installation of an oxygenated function at an allylic position has been a recurring problem. Thapsigargins are characterized by the presence of a densely oxygenated carbon skeleton. Their synthesis is complex, and the synthetic intermediates were too valuable to be wasted in trials. ${ }^{13} \mathrm{We}$ needed a model to test some of the reaction later assayed in the synthesis of thapsigargins. Valencene 1 seemed to meet our requirements: it is a commercially available sesquiterpene that presents two different double bonds, one exocyclic and another endocyclic. The different nature of these double bonds and the absence of other functional groups would simplify the analysis of the selectivity in the oxidation process.

In this work, we present our efforts to disclose the optimal conditions for the Kharasch-Sosnovsky allylic oxidation of valencene 1 (see Figure 1). In order to ensure the robustness of

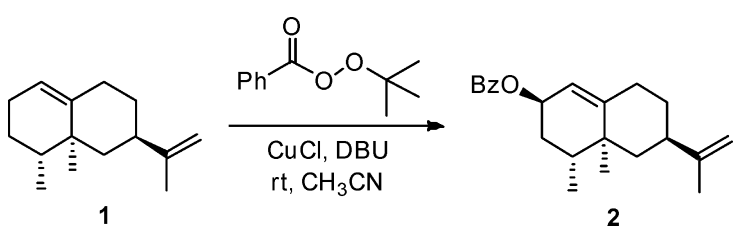

Figure 1. Kharasch-Sosnovsky oxidation of valencene.

the reaction, a design of experiments (DoE) approach has been employed. ${ }^{14}$ Although DoE has been widely adopted in fields such as analytical chemistry and chemical engineering, its use in organic synthesis in academia has not been very popular. ${ }^{15}$ Instead, the intuition of the researcher has been one of the main criteria in reaction optimization. Traditionally, an OVAT

Special Issue: Application of Design of Experiments to Process Development

Received: July 29, 2014

Published: September 19, 2014 
approach (one-variable-at-a-time) has been followed: a value for all of the variables that may affect the reaction outcome is set, and the effect on the response when only one variable is changed is studied. Nevertheless, an organic reaction is an excellent scenario for the application of DoE. The relative difficulty of the calculation methods and the need for the right choice of the experimental domain and the main variables have been the main drawbacks associated with this methodology. Nowadays, the use of modern statistic packages in conjunction with the current development of analytical techniques such as GC or LC makes possible a rapid optimization of a reaction with a smaller number of experiments.

\section{RESULTS AND DISCUSSION}

In a previous work, we have applied the simplex method to the optimization of the Kharasch-Sosnovsky oxidation of valencene $1,{ }^{16}$ obtaining the corresponding benzoate ester 2 in 55\% yield. ${ }^{17}$ We came across this reaction after some initial attempts with $\mathrm{SeO}_{2}$ and tert-butyl hydroperoxide (TBHP) as an oxidant system. We anticipated the regiochemistry of this reaction to be an issue since multiple positions were available for oxidation. Eventually, this transformation displayed poor selectivity, affording the primary and tertiary hydroxylated products resulting from the oxidation of the exocyclic double bound (at C-7 and C-13 positions), along with some overoxidation at C-13 leading to the corresponding aldehyde 5 (Figure 2).

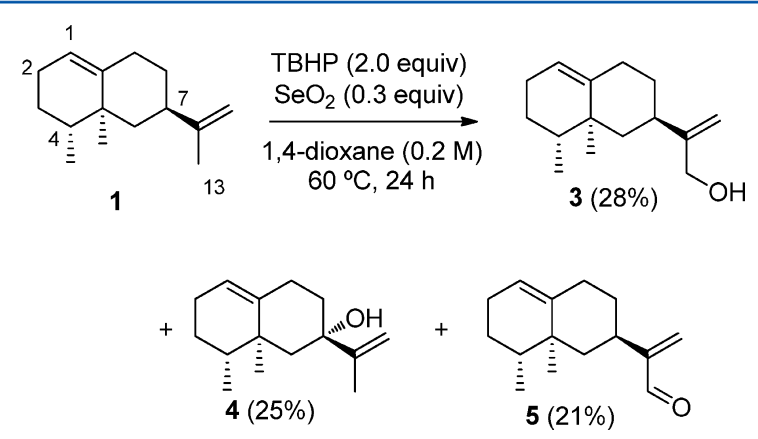

Figure 2. Allylic oxidation of valencene with $\mathrm{SeO}_{2}$ and TBHP.

As expected, $\mathrm{SeO}_{2}$ exclusively oxidized the exocyclic double bond. No insights of oxidation at C-2 was observed. Since we were more interested in the selective allylic oxidation of the endocyclic double bond, we decided to explore other possibilities as the above-mentioned Kharasch-Sosnovsky reaction for a complete optimization.

The simplex method employed in our initial study is based on a sequential optimization and can lead to good yields, but its efficiency strongly depends on the precise election of the initial conditions. Instead, the DoE approach studies the behavior of a system in an experimental domain, defined by a minimum and a maximum limit of the variables. Contrary to simplex, DoE reduces the experimental time since all experiments are run at the same time.

Response surface methodology (RSM $)^{18}$ is one of the most relevant multivariate techniques in DoE. Prior to a study by RSM and to define the experiments to be carried out, it is necessary to choose the experimental domain. This is usually done by performing a previous screening design to determine which of the experimental variables and their interactions present more significant effects. Obviously, there are plentiful variables that may affect the response of a system, and it is virtually impossible to identify and control all of them. It is therefore necessary to select carefully those variables that produce the major effects.

We decided to carry out a factorial $2^{5-1}$ screening design, considering the following variables (Table 1):

\begin{tabular}{|c|c|c|c|c|}
\hline \multicolumn{5}{|c|}{ Table 1. Levels and variables for the Factorial $2^{5-1}$ design } \\
\hline variable & symbol & low level & high level & units \\
\hline TBPB & $x_{1}$ & 1.00 & 2.50 & equiv \\
\hline $\mathrm{CuCl}$ & $x_{2}$ & 0.10 & 1.00 & equiv \\
\hline DBU & $x_{3}$ & 0.10 & 1.00 & equiv \\
\hline [valencene] & $x_{4}$ & 0.02 & 0.20 & $\mathrm{M}$ \\
\hline temperature & $x_{5}$ & 25 & 82 & ${ }^{\circ} \mathrm{C}$ \\
\hline
\end{tabular}

(i) Equivalents of TBPB: at least one equivalent of TBPB is necessary to complete the reaction. A higher amount was thought to be beneficial, and the upper limit was set to 2.50 equiv;

(ii) Concentration of valencene: in order to ensure the adequate solubility, the range of valencene concentration was $0.02-0.20 \mathrm{M}$;

(iii) Equivalents of $\mathrm{CuCl}$ : a catalytic amount of $\mathrm{CuCl}$ was desired, but the role of the $\mathrm{CuCl}$ is not entirely clear in the mechanism. A stoichiometric amount was chosen for the upper limit;

(iv) Equivalents of DBU: again, the mechanistic role of DBU is unclear, although based on previous trials amounts smaller than 1 equiv seems to be sufficient for the reaction to proceed;

(v) Temperature: the lower and the upper levels are defined by the room temperature and the boiling point of the acetonitrile.

Previous trials showed that the reaction reached the highest yield at $20 \mathrm{~h}$, and since no significant decreasing of the yield was observed, $24 \mathrm{~h}$ was selected as the reaction time for operational reasons (see Supporting Information).

This design allowed us to study the effect of five variables with 16 experiments and two replicates of the central point. ${ }^{19}$ The order of the experiments was randomized to diminish the effect of hidden variables. The experimental matrix and the yields are displayed in Table 2 .

The graph of main effects shows the tendency line for each factor (Figure 3). Every line ranges from lower to higher levels. ${ }^{20}$ From the above results, we concluded that the number of equivalents of TBPB, concentration of substrate, and temperature present a substantial impact on the response. However, the variation of the number of equivalents of $\mathrm{CuCl}$ and $\mathrm{DBU}$ did not influence appreciably on the yield. Considering that the temperature is limited by the boiling point of the solvent, it was decided to set the maximum value to $82{ }^{\circ} \mathrm{C}$ in further experiments. The effects of $\mathrm{CuCl}$ and $\mathrm{DBU}$ were slightly negative, which indicated that they should be kept at their lower levels ( 0.10 equiv of $\mathrm{CuCl}$ and 0.10 equiv of $\mathrm{DBU}$ ).

Taking into account these effects, we decided to set new conditions to obtain a response surface model. The range of the new experimental domain was redefined, increasing the equivalents of TBPB and the concentration substrate (Table 3 ).

In order to evaluate the curvature and to determine a maximum (the optimum yield), a second-order model was used. The selected design was a $3^{2}$ design (three-level full-factorial design). This type of design is not convenient for more than two variables since a large number of experiments would be required. However, for two factors, it is affordable and presents a good efficiency. ${ }^{21}$ We assumed the yield to be affected by two independent variables $\left(x_{1}\right.$ : TBPB equivalents; $x_{4}$ : valencene 1 
Table 2. Experimental matrix and results for Factorial $2^{5-1}$ design $^{a}$

\begin{tabular}{|c|c|c|c|c|c|c|}
\hline \multirow[b]{2}{*}{ run } & \multicolumn{5}{|c|}{ variables } & \multirow{2}{*}{$\frac{\text { response }}{\text { yield }^{b}}$} \\
\hline & $x_{1}$ & $x_{2}$ & $x_{3}$ & $x_{4}$ & $x_{5}$ & \\
\hline 1 & 1.00 & 1.00 & 0.10 & 0.20 & 82 & 11 \\
\hline 2 & 1.00 & 1.00 & 1.00 & 0.20 & 25 & 9 \\
\hline 3 & 1.00 & 0.10 & 1.00 & 0.02 & 25 & 0 \\
\hline 4 & 2.50 & 0.10 & 1.00 & 0.02 & 25 & 0 \\
\hline 5 & 2.50 & 1.00 & 1.00 & 0.02 & 25 & 0 \\
\hline 6 & 1.00 & 0.10 & 0.10 & 0.20 & 25 & 3 \\
\hline 7 & 2.50 & 0.10 & 1.00 & 0.20 & 25 & 5 \\
\hline 8 & 2.50 & 0.10 & 0.10 & 0.02 & 25 & 0 \\
\hline 9 & 1.75 & 0.55 & 0.55 & 0.11 & 54 & 32 \\
\hline 10 & 2.50 & 1.00 & 0.10 & 0.20 & 25 & 3 \\
\hline 11 & 1.00 & 0.10 & 0.10 & 0.02 & 82 & 7 \\
\hline 12 & 1.00 & 1.00 & 0.10 & 0.02 & 25 & 0 \\
\hline 13 & 1.00 & 1.00 & 1.00 & 0.02 & 82 & 0 \\
\hline 14 & 1.00 & 0.10 & 1.00 & 0.20 & 82 & 14 \\
\hline 15 & 2.50 & 0.10 & 0.10 & 0.20 & 82 & 80 \\
\hline 16 & 1.75 & 0.55 & 0.55 & 0.11 & 54 & 30 \\
\hline 17 & 2.50 & 1.00 & 1.00 & 0.20 & 82 & 57 \\
\hline 18 & 2.50 & 1.00 & 0.10 & 0.02 & 82 & 19 \\
\hline 19 & 1.75 & 0.55 & 0.55 & 0.11 & 54 & 29 \\
\hline
\end{tabular}

${ }^{a}$ All reactions were carried out with valencene $1(0.317 \mathrm{~mL}, 1.00$ $\mathrm{mmol}$ ), $\mathrm{TBPB}, \mathrm{CuCl}$, and $\mathrm{DBU}$ in amounts according to the table in refluxing acetonitrile for $24 \mathrm{~h} .{ }^{b}$ Determined by GC.

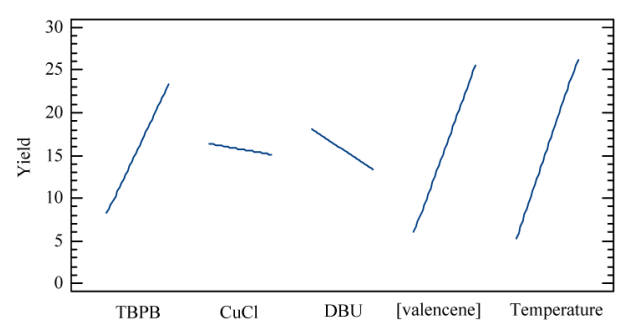

Figure 3. Main effects of the screening design.

Table 3. Levels and variables for the $3^{2}$ design

\begin{tabular}{lcccl} 
variable & symbol & low level & high level & units \\
TBPB & $x_{1}$ & 1.00 & 4.00 & equiv \\
[valencene] & $x_{4}$ & 0.01 & 0.30 & $\mathrm{M}$ \\
\hline
\end{tabular}

concentration), and the expected model would be represented by the following equation:

$$
y=\beta_{0}+\beta_{1} x_{1}+\beta_{4} x_{4}+\beta_{11} x_{1}^{2}+\beta_{44} x_{4}^{2}+\beta_{1} \beta_{4} x_{1} x_{4}
$$

where $\beta_{0}$ represents the independent term, $\beta_{1}$ and $\beta_{4}$ stand for the coefficients of the linear parameters, $\beta_{11}$ and $\beta_{44}$ are coefficients for the quadratic parameters, and $\beta_{1} \beta_{4}$ is the coefficient of the interaction parameters.

Table 4 shows the experimental matrix for the optimization of the conditions using this design, with nine experiments and three replicates of a central point.

To evaluate the goodness of the fitted model, the analysis of the variance (ANOVA) was accomplished. Therefore, an F-test to evaluate the significance of regression was made, comparing the ratio between the mean of squares of regression and the mean of squares of residuals with the Fisher distribution. When this ratio is higher than the tabulated $F$ value $\left(F_{c}\right)$, statistical significance can be concluded for the mathematical model, which
Table 4. Experimental matrix and results for $3^{2}$ design $^{a}$

\begin{tabular}{ccccc} 
& \multicolumn{2}{c}{ variables } & & response \\
\cline { 2 - 3 } run & $x_{1}$ & $x_{4}$ & yield $^{b}$ \\
1 & 4.00 & 0.10 & 23 \\
2 & 1.00 & 0.20 & 34 \\
3 & 4.00 & 0.20 & 78 \\
4 & 2.50 & 0.30 & 99 \\
5 & 2.50 & 0.20 & 78 \\
6 & 1.00 & 0.10 & 23 \\
7 & 2.50 & 0.20 & 66 \\
8 & 2.50 & 0.10 & 32 \\
9 & 4.00 & 0.30 & 91 \\
10 & 2.50 & 0.20 & 51 \\
11 & 2.50 & 0.20 & 85 \\
12 & 1.00 & 0.30 & 56
\end{tabular}

${ }^{a}$ All reactions were carried out with valencene $\mathbf{1}(0.317 \mathrm{~mL}, 1.00$ $\mathrm{mmol}), \mathrm{TBPB}, \mathrm{CuCl}(10 \mathrm{mg}, 0.10 \mathrm{mmol}, 0.10$ equiv), and $\mathrm{DBU}$ $(0.015 \mathrm{~mL}, 0.10 \mathrm{mmol}, 0.10$ equiv) in refluxing acetonitrile for $24 \mathrm{~h}$. ${ }^{b}$ Determined by GC.

Table 5. ANOVA results of the quadratic model for the yield of benzoyloxyvalencene $2^{a}$

\begin{tabular}{lccccc}
\multicolumn{1}{c}{ source } & $\begin{array}{c}\text { sum of } \\
\text { squares }\end{array}$ & $\begin{array}{c}\text { degree of } \\
\text { freedom }\end{array}$ & $\begin{array}{c}\text { mean } \\
\text { squares }\end{array}$ & $\begin{array}{c}F \\
\text { values }\end{array}$ & $\begin{array}{c}\text { critic } F \text { values } \\
\left(F_{\mathrm{c}}\right)\end{array}$ \\
\hline model & 7252.25 & 6 & 1192.11 & 5.89 & 4.95 \\
residual & 1011.60 & 5 & 202.32 & & \\
lack of fit & 445.60 & 2 & 172.80 & 0.78 & 9.55 \\
pure error & 666.00 & 3 & 222.00 & & \\
total & 8164.25 & 11 & & & \\
${ }^{a} R^{2}=0.8761 ; R=0.9360 ;$ & $\%$ adjustment $=93.60$. & \\
\hline
\end{tabular}

means that it is well-fitted to the experimental data. As shown in Table 5, a model $F$ value of 5.89 for the yield of benzoyloxyvalencene 2 indicates that the model is significant. On the other hand, the lack of fit is not significant relative to pure error since its $F$ value is 0.78 , lower than $F_{\mathcal{c}}$, which also accounts for the goodness of the model. The model explains the $88 \%$ of the variability in the yield of benzoyloxyvalencene 2 , according to the obtained value for the $R^{2}$ coefficient $(0.8761)$.

To identify the constants and coefficients for each variable, multiple regression analyses were performed by using the experimental data (Table 6).

Table 6. Regression coefficients and $p$ values for benzoyloxyvalencene 2 yield

\begin{tabular}{lrr} 
variables & coefficients & $p$ values \\
constant & -46.0833 & \\
$x_{1}$ & 32.3889 & 0.0476 \\
$x_{4}$ & 370.8330 & 0.0018 \\
$x_{1}{ }^{2}$ & -7.0556 & 0.0929 \\
$x_{4}$ & -587.5000 & 0.2264 \\
$x_{1} x_{4}$ & 58.3333 & 0.4879 \\
\hline
\end{tabular}

Fitting the constants and coefficients, the equation that represents the chosen model is

$$
\begin{aligned}
y= & -46.0833+32.3889 x_{1}+370.8330 x_{4}-7.0556 x_{1}^{2} \\
& -587.5000 x_{4}^{2}+58.3333 x_{1} x_{4}
\end{aligned}
$$


Values of $p<0.05$ indicate significant model terms. Both variables, TBPB $(p=0.0476)$ and concentration of valencene 1 $(p=0.0018)$ had a significant linear effect on the benzoyloxyvalencene 2 yield. Pareto plot shows these conclusions about the statistical significance of the effects. The vertical line in this graph indicates a level of $95 \%$ confidence. Horizontal bars that cross this line represent variables with greater influence on the yield of the reaction for that level of confidence (Figure 4).

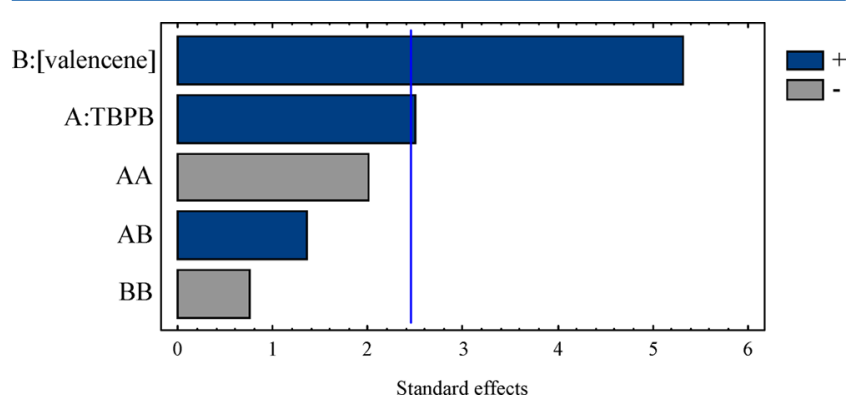

Figure 4. Pareto plot of $3^{2}$ design for benzoyloxyvalencene 2 .

An evaluation of the adequacy of the model was made by using the residuals from the least-squares fit. The normal probability plot of the residuals (Figure 5) indicates that normality assumptions are satisfactory since the residual plots cluster around the diagonal line.

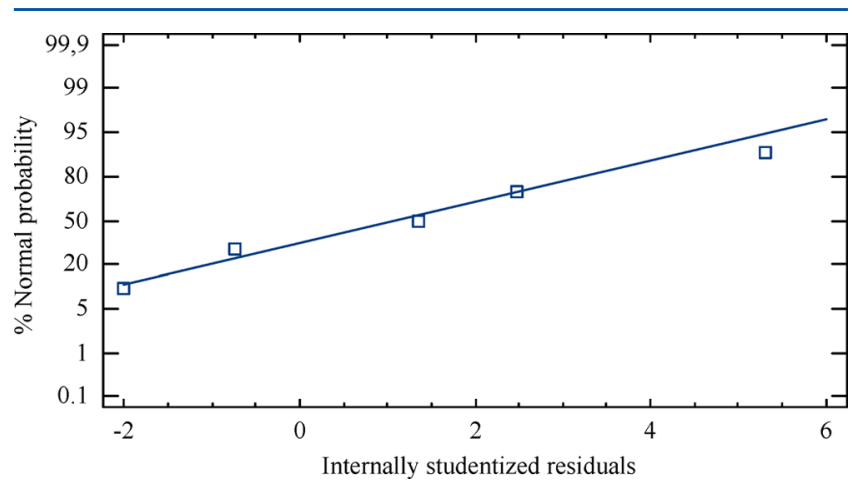

Figure 5. Normal probability of internally residuals.

Figure 6 represents the plot of residuals versus predicted responses. The residuals are distributed in a random fashion without any trend. These results imply that a maximum approximation on the relationship between variables and responses is provided by the obtained model.

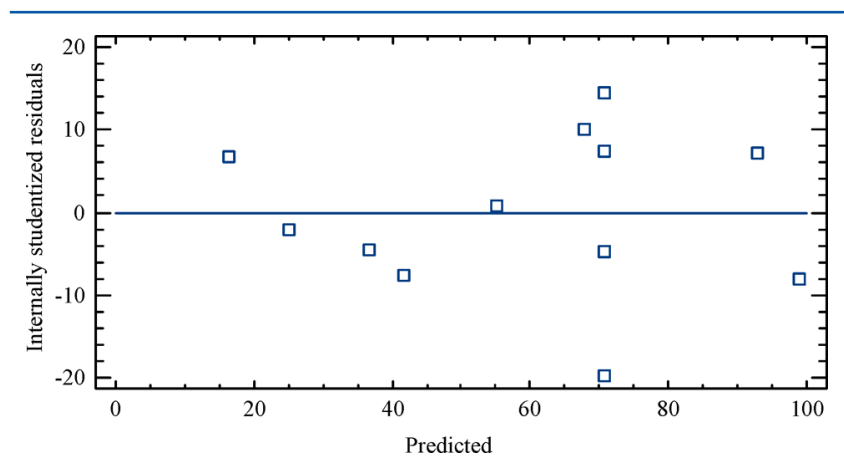

Figure 6. Plot of internally studentized residuals vs predicted for the yield of benzoyloxyvalencene 2 .
It is possible to calculate the coordinates of the maximum (optimum yield) through the first derivate of the mathematical function. The visualization of the predicted model equation can be obtained by the surface response plot (Figure 7). The

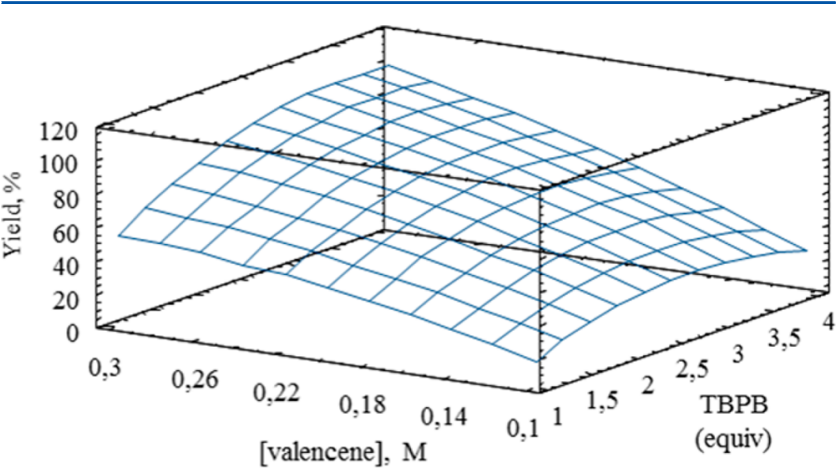

Figure 7. Response surface estimated for yield of benzoyloxyvalencene 2.

response surface as a function of the independent variables within the experimental range was generated by using the empirical model presented in eq 2 . The plot indicates that the optimum yield was localized when we used 2.50 equiv of TBPB and a $0.30 \mathrm{M}$ concentration of valencene 1 .

After optimization by using the quadratic model, the predicted optimal conditions within the experimental domain were 3.54 equiv of TBPB and $0.30 \mathrm{M}$ concentration. This set of conditions affords a predicted maximum yield of benzoyloxyvalencene 2 of $100.48 \%$

Finally, we decided to run an experiment with the predicted optimum conditions. This confirmation reaction led to $84 \%$ yield instead of the predicted $100 \%$. However, a $99 \%$ yield was achieved experimentally with a lower amount of TBPB as shown in Table 7.

Table 7. Yields obtained for benzoyloxyvalencene 2 by the Kharasch-Sosnovsky reaction

\begin{tabular}{lccc}
\multicolumn{1}{c}{ conditions } & TBPB (equiv) & [valencene] (M) & yield \\
experimental $^{a}$ & 2.50 & 0.30 & $99^{b}$ \\
predicted $^{a}$ & 3.54 & 0.30 & 100 \\
confirmed $^{a}$ & 3.54 & 0.30 & $84^{b}$
\end{tabular}

${ }^{a}$ All reactions were carried out with valencene $1(0.317 \mathrm{~mL}, 1.00$ $\mathrm{mmol}$ ), TBPB, $\mathrm{CuCl}$ ( $10 \mathrm{mg}, 0.10 \mathrm{mmol}, 0.10$ equiv), DBU (0.015 $\mathrm{mL}, 0.10 \mathrm{mmol}, 0.10$ equiv) in refluxing acetonitrile for $24 \mathrm{~h}$. ${ }^{b}$ Determined by GC.

There is a discrepancy of $16 \%$ between the predicted and the confirmed experiments in Table 7 . This result can be explained by considering that the Kharasch-Sosnovsky reaction takes place through a complex mechanism involving multiple species, including copper in different oxidation states and the formation of different radicals. ${ }^{6 c}$ It is not surprising to find these discrepancies in such a complex organic reaction system. Anyhow, the increase in the yield of the reaction to $84 \%$ (found by the model optimization) and to 99\% (found through the experimental design) has demonstrated the superior potential of the DoE approach compared to the classical OVAT methodology. ${ }^{22}$ 


\section{CONCLUSION}

The optimization of the Kharasch-Sosnovsky allylic oxidation of valencene $\mathbf{1}$ has been accomplished. Different variables were selected for DoE and RSM designs and the reaction yield as the response was improved from 55 to $99 \%$. According to the ANOVA analysis, the fitted model accounts for the behavior of the studied system. This is an example of the rapid and efficient optimization of a mechanistically unclear and complex chemical system and can serve as a guide for modeling organic reactions in academia.

\section{ASSOCIATED CONTENT}

\section{S Supporting Information}

General considerations, optimized procedure for the KharaschSosnovsky oxidation of valencene, and spectral data of 2benzoyloxyvalencene. This material is available free of charge via the Internet at http://pubs.acs.org.

\section{AUTHOR INFORMATION}

\section{Corresponding Author}

*Tel.: +34-956012762. E-mail: francisco.guerra@uca.es.

\section{Notes}

The authors declare no competing financial interest.

\section{ACKNOWLEDGMENTS}

This research was supported by Junta de Andalucía (FQM-169). A.L.G.-C. and R.M.-B. thank the Spanish Ministry of Education, Culture and Sport for a fellowship. L. P. R. acknowledges the Fulbright Foundation for a fellowship.

\section{REFERENCES}

(1) (a) White, M. C. Synlett 2012, 23, 2746-2748. (b) Wencel-Delord, J.; Dröge, T.; Liu, F.; Glorius, F. Chem. Soc. Rev. 2011, 40, 4740-4761. (c) Bergman, R. G. Nature 2007, 446, 391-393. (d) Shilov, A. E.; Shul'pin, G. B. Chem. Rev. 1997, 97, 2879-2932.

(2) (a) Ammann, S. E.; Rice, G. T.; White, M. C. J. Am. Chem. Soc. 2014, 136, 10834-10837. (b) Fraunhoffer, K. J.; Prabagaran, N.; Sirois, L. E.; White, M. C. J. Am. Chem. Soc. 2006, 128, 9032-9033. (c) Covell, D. J.; Vermeulen, N. A.; Labenz, N. A.; White, M. C. Angew. Chem., Int. Ed. 2006, 118, 8397-8400. (d) Fraunhoffer, K. J.; Bachovchin, D. A.; White, M. C. Org. Lett. 2005, 7, 223-226.

(3) Sharma, A.; Hartwig, J. F. J. Am. Chem. Soc. 2013, 135, 1798317989.

(4) Weidmann, V.; Maison, W. Synthesis 2013, 45, 2201-2221.

(5) Sharpless, K. B.; Lauer, R. F. J. Am. Chem. Soc. 1972, 94, 71547155

(6) (a) Andrus, M. B.; Lashley, J. C. Tetrahedron 2002, 58, 845-866. (b) Eames, J.; Watkinson, W. Angew. Chem., Int. Ed. 2001, 40, 35673571. (c) Mayoral, J. A.; Rodríguez-Rodríguez, S.; Salvatella, L. Chem.Eur. J. 2008, 14, 9274-9285.

(7) (a) Kharasch, M. S.; Sosnovsky, G.; Yang, N. C. J. Am. Chem. Soc. 1959, 81, 5819-5824. (b) Kharasch, M. S.; Sosnovsky, G. J. Am. Chem. Soc. 1958, 80, 756 .

(8) Ramalingan, B.; Neuburger, M.; Pfaltz, A. Synthesis 2007, 572-582. (9) Kawasaki, K.; Tsumura, S.; Katsuki, T. Synlett 1995, 1245-1246. (10) (a) Andrus, M. B.; Zhou, Z. J. Am. Chem. Soc. 2002, 124, 88068807. (b) Andrus, M. B.; Asgari, D. Tetrahedron 2000, 56, 5775-5780. (11) Newhouse, T.; Baran, P. S. Angew. Chem., Int. Ed. 2011, 50, 33623374.

(12) (a) Lytton, J.; Westlin, M.; Hanley, M. R. J. Biol. Chem. 1991, 266, 17067-17071. (b) Inesi, G.; Sagara, Y. Arch. Biochem. Biophys. 1992, 298, 313-317. (c) Christensen, S. B.; Andersen, A.; Smitt, U. W. Prog. Chem. Org. Nat. Prod. 1997, 71, 130-167. (d) Treiman, M.; Carpersen, C.; Christensen, S. B. Trends Pharmacol. Sci. 1998, 19, 131-135.

(e) Christensen, S. B.; Andersen, A.; Kromann, H.; Treiman, M.;
Tombal, B.; Denmeade, S. R.; Isaacs, J. T. Biorg. Med. Chem. 1999, 7, 1273-1280. (f) Jacobsen, C. M.; Denmeade, S. R.; Isaacs, J. T.; Gady, A.; Olsen, C. E.; Christensen, S. B. J. Med. Chem. 2001, 44, 4696-4703.

(13) (a) Ball, M.; Andrews, S. P.; Wierschem, F.; Cleator, E.; Smith, M. D.; Ley, S. V. Org. Lett. 2007, 9, 663-666. (b) Manzano, F. L.; Guerra, F. M.; Moreno-Dorado, F. J.; Jorge, D. Z.; Massanet, G. M. Org. Lett. 2006, 8, 2879-2882. (c) Ley, S. V.; Antonello, A.; Balskus, E. P.; Booth, D. T.; Christensen, S. B.; Cleator, E.; Gold, H.; Högenauer, K.; Hünger, U.; Myers, R. M.; Oliver, S. F.; Simic, O.; Smith, M. D.; Søhoel, H.; Wooldford, A. J. A. Proc. Natl. Acad. Sci. U.S.A. 2004, 101, 12073-12078. (d) Oliver, S. F.; Högenauer, K.; Simic, O.; Antonello, A.; Smith, M. D.; Ley, S. V. Angew. Chem., Int. Ed. 2003, 42, 5996-6000.

(14) For a general introduction to experimental design in chemistry, see: (a) Weissman, S. A.; Anderson, N. G. Org. Process Res. Dev. 2014, DOI: 10.1021/op500169m. (b) Leardy, R. Anal. Quim. Acta 2009, 652, 161-172. (c) Carlson, R.; Carlson, J. E. Design and Optimization in Organic Synthesis. In Data Handling in Science and Technology; Elsevier: Amsterdam, 2005. (d) Araujo, P. W.; Brereton, R. G. TrAC, Trends Anal. Chem. 1996, 15, 26-31. (e) Araujo, P. W.; Brereton, R. G. TrAC, Trends Anal. Chem. 1996, 15, 63-70.

(15) (a) Marín-Barrios, R.; García-Cabeza, A. L.; Moreno-Dorado, F. J.; Guerra, F. M.; Massanet, G. M. J. Org. Chem. 2014, 79, 6501-6509. (b) García-Cabeza, A. L.; Marín-Barrios, R.; Moreno-Dorado, F. J.; Ortega, M. J.; Massanet, G. M.; Guerra, F. M. Org. Lett. 2014, 16, 15981601. (c) García-Cabeza, A. L.; Marín-Barrios, R.; Azarken, R.; MorenoDorado, F. J.; Ortega, M. J.; Vidal, H.; Gatica, J. M.; Massanet, G. M.; Guerra, F. M. Eur. J. Org. Chem. 2013, 36, 8307-8314. (d) Carlons, R. Chemometr. Intell. Lab. 2004, 73, 151-166.

(16) (a) Betteridge, D.; Wade, A. P.; Howard, A. G. Talanta 1985, 32, 709-722. (b) Chubb, F. L.; Edward, J. T.; Wong, S. C. J. Org. Chem. 1980, 45, 2315-2320.

(17) Marín-Barrios, R.; Guerra, F. M.; García-Cabeza, A. L.; MorenoDorado, F. J.; Massanet, G. M. Tetrahedron 2012, 68, 1105-1108.

(18) (a) Rosenthal, I.; Wolfram, E.; Peter, S.; Meier, B. J. Nat. Prod. 2014, 77, 489-496. (b) Fan, J.; Yi, C.; Lan, X.; Yang, B. Org. Process Res. Dev. 2013, 17, 368-374. (c) Bezerra, M. A.; Santelli, R. E.; Oliveira, E. P.; Villar, L. S.; Escaleira, L. A. Talanta 2008, 76, 965-977.

(19) It is possible to perform an initial screening design with a lower number of runs, but 16 reactions is an affordable number of experiments, and the obtained data would display less aliasing.

(20) Although the results presented some curvature, second-order effects could be confounded in the screening design. For this reason, curved graphics for the main effects are not displayed.

(21) In the case that a higher number of variables had been implied, other alternatives should have been considered. A central composite design would have been an appropriate choice.

(22) New refinements could lead to a more accurate model, but a $84 \%$ yield is good enough for synthetic purposes. In addition, the screening afforded conditions in which $99 \%$ yield was obtained. This means that although it cannot be concluded that the model is robust, the optimization process was successful. 